\title{
Dynamic Interactions between Autophagosomes and Lipid Droplets in Chlamydomonas reinhardtii
}

\author{
Quynh-Giao Tran ${ }^{1,2}$, Hyang Ran Yoon ${ }^{3}$, Kichul Cho ${ }^{4}$, Seon-Jin Lee ${ }^{5}$, José L. Crespo ${ }^{6} \oplus$, \\ Rishiram Ramanan ${ }^{7}$ and Hee-Sik Kim 1,2,* \\ 1 Cell Factory Research Center, Korea Research Institute of Bioscience and Biotechnology (KRIBB), \\ Daejeon 34141, Korea \\ 2 Department of Environmental Biotechnology, KRIBB School of Biotechnology, University of Science and \\ Technology, Daejeon 34113, Korea \\ 3 Immunotherapy Convergence Research Center, KRIBB, Daejeon 34141, Korea \\ 4 Environmental Safety Group, Korea Institute of Science and Technology (KIST) Europe, Campus E 7.1, \\ 66123 Saarbrücken, Germany \\ 5 Environmental Disease Research Center, KRIBB, Daejeon 34141, Korea \\ 6 Instituto de Bioquímica Vegetal y Fotosíntesis, CSIC-Universidad de Sevilla, 41092 Sevilla, Spain \\ 7 Sustainable Resources Laboratory, Department of Environmental Science, Central University of Kerala, \\ Kasaragod 671316, Kerala, India \\ * Correspondence: hkim@kribb.re.kr; Tel.: +82-42-860-4326
}

Received: 14 August 2019; Accepted: 26 August 2019; Published: 28 August 2019

\begin{abstract}
Autophagy is a highly conserved catabolic process in eukaryotic cells by which waste cellular components are recycled to maintain growth in both favorable and stress conditions. Autophagy has been linked to lipid metabolism in microalgae; however, the mechanism underlying this interaction remains unclear. In this study, transgenic Chlamydomonas reinhardtii cells that stably express the red fluorescent protein (mCherry) tagged-ATG8 as an autophagy marker were established. By using this tool, we were able to follow the autophagy process in live microalgal cells under various conditions. Live-cell and transmission electron microscopy (TEM) imaging revealed physical contacts between lipid droplets and autophagic structures during the early stage of nitrogen starvation, while fusion of these two organelles was observed in prolonged nutritional deficiency, suggesting that an autophagy-related pathway might be involved in lipid droplet turnover in this alga. Our results thus shed light on the interplay between autophagy and lipid metabolism in C. reinhardtii, and this autophagy marker would be a valuable asset for further investigations on autophagic processes in microalgae.
\end{abstract}

Keywords: autophagy marker; Chlamydomonas; chloroquine; lipid droplet; mCherry-ATG8; microalgal lipophagy

\section{Introduction}

Microalgae are plant-like organisms that inhabit freshwater, inter-tidal, marine, and extreme environments. Microalgae are known to accumulate valuable products such as lipids, starch, and carotenoids in response to unfavorable conditions [1]. Thus, treatment of algae with nutrient deprivation, high salinity, extreme temperatures, or high irradiance have become essential strategies for enhancing commercial production of microalgal metabolites [1-4]. It has been shown that upon exposure to external stressors, eukaryotic cells rapidly trigger a series of systemic responses, which are controlled by autophagy among other factors, to promote survival $[5,6]$. More specifically, autophagy helps with cleaning and recycling cellular "waste" including protein aggregates and damaged organelles caused by a range of abiotic and biotic stresses in many organisms [5,7-9]. Autophagy has been 
intensively studied in animals and yeasts over the last few decades; however, challenges to expand this research area in microalgae still remain, mostly due to the lack of monitoring tools [9].

In recent years, there has been significant progress in exploring the role of autophagy in the unicellular model C. reinhardtii [10-18]. For instance, two studies demonstrated that inhibition of autophagic process by treatment with autophagy inhibitors including concanamycin A, bafilomycin A1, and wortmannin reduced the number of lipid droplets accumulated in C. reinhardtii cells under nutrient starvation $[15,16]$. These results suggested that autophagy might be involved in the biogenesis of lipid droplets in this alga. On the contrary, the role of autophagy in lipid degradation was demonstrated in the green microalga Auxenochlorella protothecoides when the cells were transferred from heterotrophic to autotrophic growth conditions [19]. How autophagy regulates stress responses in microalgae and how it interacts with algal lipid metabolism in stress conditions remain open questions. A better understanding of this interaction could provide insights to advance the production of biofuel precursors and other valuable metabolites in microalgae.

Autophagic activity can be assessed by observing autophagy-related structures and analyzing the abundance/modification of autophagy-related proteins [20]. Among these proteins, the autophagy-related protein 8 (ATG8) plays a critical role in the formation and maturation of autophagosome in eukaryotic organisms [21]. In C. reinhardtii, ATG8 contains an extended 14-amino-acid sequence after the conserved glycine residue (Gly120) at its C-terminus [9]. The nascent ATG8 protein is cleaved at its Gly120 residue by the ATG4 protease to form a cytosolic unconjugated ATG8 protein. Upon autophagy induction, the unconjugated ATG8 becomes conjugated/lipidated to the phospholipid phosphatidylethanolamine (PE) to form ATG8-PE by the action of E1- and E2-like enzymes [9]. Importantly, the ATG8-PE remains associated with autophagosome from the early formation to completed fusion with the lysosome/lytic vacuole, making it a specific marker for monitoring autophagy in vivo [22]. A specific ATG8 antibody has been developed in C. reinhardtii, and the lipidation status of this protein can be easily assessed by Western blot analysis [11]. Furthermore, cellular distribution of autophagosomes can be visualized by TEM imaging and immunolocalization assay using ATG8 antibody, although these approaches may require specialized skills and equipment [9]. These methods were the only tools used for setting up the basic knowledge of autophagy-related mechanisms in this alga to date. However, monitoring of autophagic flux and co-localization studies in living algal cells remained entirely unexplored [9].

In this study, we developed C. reinhardtii transgenic lines expressing the red fluorescent protein ( $m$ Cherry)-ATG8 and investigated the formation of autophagosomes in live algal cells under different conditions. The effect of chloroquine (CQ), an inexpensive lysosomotropic agent, on lytic vacuolar activity and autophagic flux was also examined. In addition, Western blot and TEM analyses were carried out in order to validate autophagic activity in the mutants. By using live-cell imaging, we observed physical interactions between mCherry-labeled autophagosomes and lipid droplets in this green alga under nitrogen starvation. To our knowledge, this provides the first visual evidence for lipid droplet-autophagosome interaction in microalgae.

\section{Materials and Methods}

\subsection{Microalgal Cultivation}

C. reinhardtii wild-type strain CC-124 [137c] was grown in Tris-acetate phosphate (TAP) medium [23], in $500 \mathrm{~mL}$ conical flasks under continuous illumination of $50 \pm 10 \mu \mathrm{mol} \mathrm{m} \mathrm{m}^{-2} \mathrm{~s}^{-1}$ at $25^{\circ} \mathrm{C}$, with constant shaking at $90 \mathrm{rpm}$. When required, a solid medium was prepared by adding $15 \mathrm{~g}$ bacto agar per $1 \mathrm{~L}$ TAP medium. For nitrogen starvation, cells in exponential phase (approximate cell density $1 \times 10^{6}$ cells $\mathrm{mL}^{-1}$ ) were harvested by centrifugation (2000× $g$ for $5 \mathrm{~min}$ ). Cell pellet was washed once in nitrogen-free medium (TAP-N) before resuspension in TAP-N at the same cell density. For selection of transformants, paromomycin (Sigma-Aldrich, St. Louis, MO, USA) was added to liquid or agar solidified TAP medium at concentration of $25 \mu \mathrm{g} \mathrm{mL}-1$. 


\subsection{Vector Construction}

To generate $m$ Cherry-ATG8 fusion construct, the codon-optimized sequence of $m$ Cherry gene (removed the stop codon) was PCR amplified from the pBR9 mCherry Cr plasmid [24] and cloned into the pET-28a(+) cloning vector as a XhoI/HindIII fragment in front of the CrATG8 gene. The RBCS2 intron1 obtained from the pChlamiRNA3int plasmid (Chlamydomonas Resource Center, St. Paul, MN, USA) was cloned as a NdeI/XhoI fragment in front of the mCherry-ATG8 sequence. Then, the full set (RBCS2 intron1-mCherry-ATG8) was cloned back into pChlamiRNA3int to create the pChl-mCherry-ATG8 expression vector (Figure 1).

\subsection{Generation of mCherry-ATG8 Transgenic Lines}

Wild-type cells were transformed by electroporation with GeneArt ${ }^{\circledR}$ MAX Efficiency ${ }^{\circledR}$ Transformation Reagent for Algae protocol and reagent (Invitrogen, Carlsbad, CA, USA). In brief, cells were grown to $1 \times 10^{6}$ cells $\mathrm{mL}^{-1}$ in TAP medium as described. Cells were harvested by centrifugation at $2000 \times g$ for $5 \mathrm{~min}$ and washed twice with transformation reagent. Cell pellet was resuspended in transformation reagent to a final concentration of $2 \times 10^{8}$ cells $\mathrm{mL}^{-1}$. A total of $1 \mu \mathrm{g}$ of linearized plasmid was incubated with $250 \mu \mathrm{L}$ of cells for $5 \mathrm{~min}$ on ice. The cell-plasmid mix was then transferred into an ice-cold $0.4 \mathrm{~cm}$-gap cuvette (Bio-Rad, Hercules, CA, USA). Electroporation was performed using the Gene Pulser Xcell ${ }^{\mathrm{TM}}$ Total System (Bio-Rad, Hercules, CA, USA) with the following conditions: Resistance of $800 \Omega$, capacity of $50 \mu \mathrm{F}$, field strength of $1.25 \mathrm{kV} \mathrm{cm}^{-1}$, and pulse duration of $30 \pm 2 \mathrm{~ms}$. Cells were recovered for 14-16 h in $10 \mathrm{~mL}$ of TAP supplemented with $40 \mathrm{mM}$ sucrose under dim light at $25^{\circ} \mathrm{C}$, with gentle shaking at $50 \mathrm{rpm}$ and then plated on TAP agar plates supplemented with paromomycin. After 7 days of incubation, single green colonies were transferred to liquid medium containing $25 \mu \mathrm{g} \mathrm{mL}^{-1}$ paromomycin. Stable transformants were obtained after several rounds of selection and subjected to further analyses.

\subsection{Protein Isolation and Western Blot Analysis}

Total cell lysates were prepared with RIPA buffer (100 mM Tris-HCl pH 8.0, 5 mM EDTA, 50 mM $\mathrm{NaCl}, 50 \mathrm{mM} \beta$-glycerophosphate, $50 \mathrm{mM} \mathrm{NaF}, 0.1 \mathrm{mM} \mathrm{Na} \mathrm{VO}_{4}, 1 \mathrm{mM}$ PMSF, 0.5\% NP-40, 0.5\% sodium deoxycholate, supplemented with $10 \mu \mathrm{L} \mathrm{mL}^{-1}$ Protease Inhibitor Cocktail (Sigma-Aldrich, St. Louis, MO, USA) before use. Same amounts of total protein were denatured by incubation with $5 \times$ SDS sample buffer for $5 \mathrm{~min}$ in boiling water bath. A total of $30 \mu \mathrm{g}$ of protein per sample was separated on 15\% SDS-PAGE gels at 110 volts and transferred to PVDF membrane (Bio-Rad, Hercules, CA, USA) at $350 \mathrm{~mA}$ for $1 \mathrm{~h}$. The anti-CrATG8 polyclonal antibody was kindly provided by Dr. José L. Crespo (Universidad de Sevilla). For Western blot analysis, anti-CrATG8 antibody was diluted 1:2000 in Tris-buffered saline containing 0.1\% Tween 20 (TBST buffer) and 5\% (w/v) skim milk. Anti-rabbit (Abcam, Cambridge, UK) secondary antibodies were diluted 1:10,000 in the same buffer and used to detect ATG8 protein. The Clarity ${ }^{\mathrm{TM}}$ Western ECL Substrate (Bio-Rad, Hercules, CA, USA) was used to generate signals and images were captured by Bio-Rad ChemiDoc ${ }^{\mathrm{TM}} \mathrm{MP}$ System.

\subsection{Expression Analysis by Real-Time RT-PCR}

Cell culture was harvested and cell pellet was snap frozen with liquid nitrogen. Total RNA was extracted using TRIzol ${ }^{\circledR}$ Reagent (Ambion, Austin, TX, USA) and purified with QIAGEN RNeasy Mini Kit in accordance with the manufacturer's protocol. Genomic DNA contamination was eliminated from total RNA sample using RQ1 RNase-Free DNase kit (Promega, Madison, WI, USA), and RNA purity and quantity were checked with the NanoPhotometer ${ }^{\circledR}$ P360 device (Implen, Munich, Germany). A total of $500 \mathrm{ng}$ of total RNA was converted to first-strand cDNA with oligo(dT) primer using GoScript ${ }^{\mathrm{TM}}$ Reverse Transcription System (Promega, Madison, WI, USA) in a $20 \mu \mathrm{L}$ reaction. The resulting cDNA was used as template for real-time RT-PCR using $\mathrm{iQ}^{\mathrm{TM}}$ SYBR ${ }^{\circledR}$ Green Supermix (Bio-Rad, Hercules, CA, USA) with the following primers: mCherry_F (5'-ACATCAAGCTGGACATCACC-3') and mCherry_R 
(5'-CTTGTACAGCTCGTCCATGC-3'). A total of $10 \mu \mathrm{L}$ of each RT-PCR product was resolved by electrophoresis on a $1 \%(w / v)$ agarose gel, and the gel image was captured with a Kodak Gel Logic 100 Digital Imaging System (Kodak, Rochester, NY, USA).

\subsection{Confocal Microscopic Analysis}

For lytic vacuole staining, exponential phase cells treated with autophagic stimuli (rapamycin or nitrogen depletion) were harvested by centrifugation at $2000 \times \mathrm{g}$ for $5 \mathrm{~min}$ and placed in $1 \mathrm{~mL}$ of the same fresh medium. LysoSensor Green DND-189 (stock $1 \mathrm{mM}$ in DMSO) (Life Technologies, Carlsbad, CA, USA) was then added to the sample at the final concentration of $5 \mu \mathrm{M}$ and incubated for $30 \mathrm{~min}$ in the dark at $37^{\circ} \mathrm{C}$ according to the manufacturer's protocol. At the end of the incubation, cells were directly observed under the confocal facility without further treatment. For lipid droplet staining, cells were harvested and stained with $1 \mu \mathrm{g} \mathrm{mL}^{-1}$ BODIPY 505/515 (Invitrogen, Carlsbad, CA, USA) as described previously [17].

Images were obtained by using a Zeiss LSM510 meta-laser scanning confocal microscope (Carl Zeiss AG, Oberkochen, Germany) fitted with 100× objectives and equipped with Nikon camera. Excitation/emission maxima of $587 / 610 \mathrm{~nm}$ for mCherry, $655 / 667 \mathrm{~nm}$ for chlorophyll $a, 448 / 505 \mathrm{~nm}$ for LysoSensor Green DND-189, and 505/515 nm for BODIPY 505/515 were used to acquire fluorescence images. Images were processed using Zeiss LSM510 software (Carl Zeiss AG, Oberkochen, Germany) and quantified using Image software (NIH, Bethesda, MD, USA) when necessary.

\subsection{Transmission Electron Microscopy (TEM) Analysis}

The cells were fixed in $2.5 \%$ paraformaldehyde-glutaraldehyde mixture buffered with $0.1 \mathrm{M}$ phosphate ( $\mathrm{pH} 7.2)$ for $2 \mathrm{~h}$, post fixed in 1\% osmium tetroxide in the same buffer for $1 \mathrm{~h}$, dehydrated in graded ethanol and propylene oxide, and embedded in Epon-812. Ultra-thin sections, made by ULTRACUT E (Leica, Wetzlar, Germany) ultramicrotome, were stained with uranyl acetate and lead citrate and examined under CM 20 (Philips, Amsterdam, The Netherlands) electron microscope.

\subsection{Flow Cytometry Analysis}

Flow cytometry analysis was performed using the BD FACS Aria ${ }^{\mathrm{TM}}$ Cell Sorters (BD Biosciences, San Jose, CA, USA) equipped with $488 \mathrm{~nm}$ (blue) and $633 \mathrm{~nm}$ (red) lasers. Data was analyzed with FlowJo ${ }^{\mathrm{TM}}$ software version 7.6.5 (FlowJo LLC, Ashland, OR, USA). A minimum of 50,000 algal cells per sample were recorded for fluorescence analysis. Experiments were repeated three times and mean values of fluorescence intensity are shown.

\subsection{Quantification of Autophagic Structures}

In order to quantify the number and size of mCherry-labeled autophagic structures, confocal images were processed and analyzed using ImageJ software (version 1.46, NIH, Bethesda, MD, USA). The number and size of autophagic structures per cell were automatically measured using the Analyze Particles function in ImageJ after setting the threshold (only particles larger than 5 pixels were included). In Figure 3, the lipid droplets positive with mCherry-ATG8 were defined as structures which labeled with both red and green fluorescence. Co-localization analysis was done using the co-localization plugin in Image (http://imagej.net/mbf/colour_analysis.htm) and the number of co-localized structures were counted per cell. For all experiments, approximately 50-80 cells per condition were analyzed in at least five independent confocal images per condition.

\subsection{Statistical Analyses}

All experiments were performed in triplicate and data are presented as the means \pm standard deviation (SD). Statistical analyses were done using Origin Pro 9.0 software (OriginLab, Northampton, MA, USA). One-way ANOVA and subsequent Tukey's post-hoc $t$ test or Mann-Whitney U test was used to analyze the statistical significance $\left({ }^{* *}, p<0.01\right)$ of the data. 
A

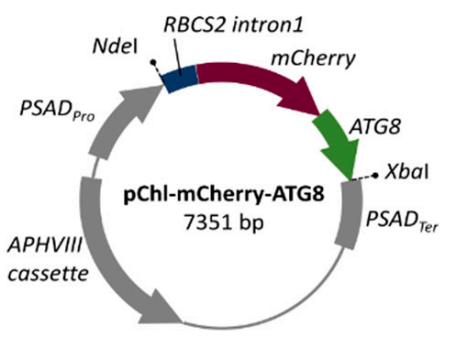

C

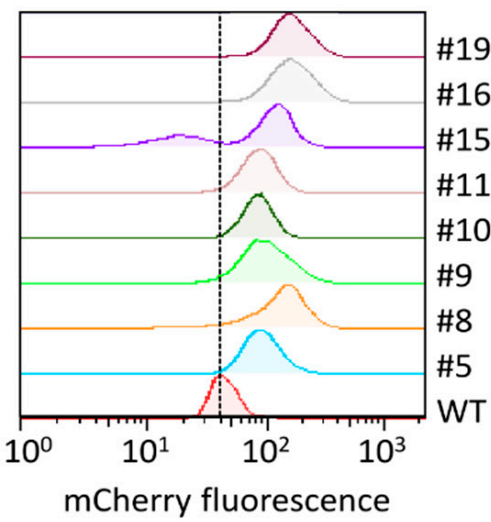

B
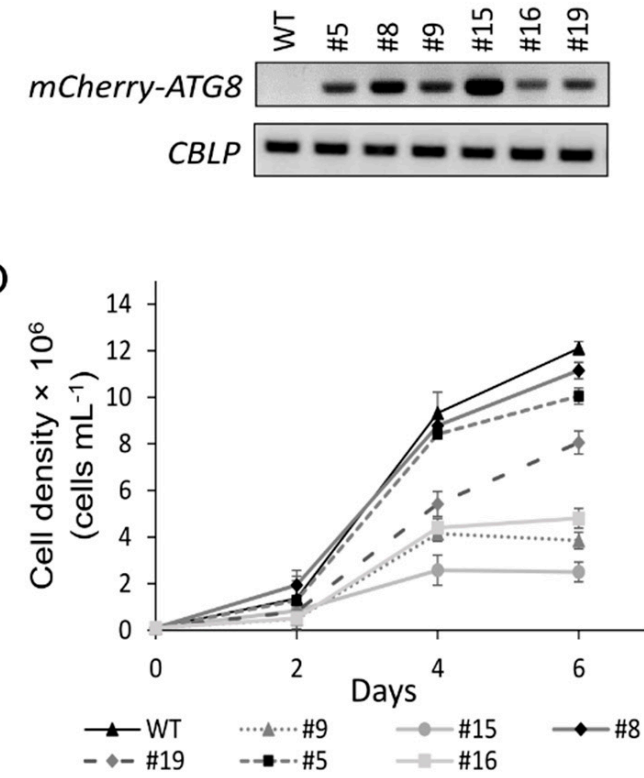

$E$

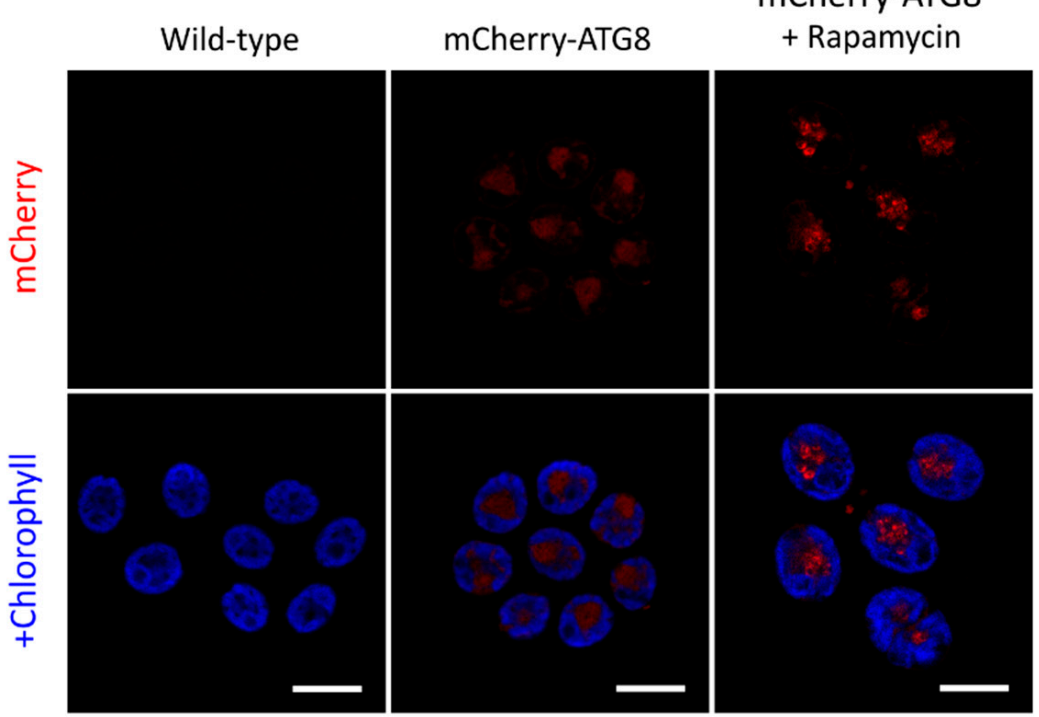

Figure 1. Generation of $C$. reinhardtii transgenic lines expressing the red fluorescent protein (mCherry)-ATG8. (A) Schematic drawing of the pChl-mCherry-ATG8 vector for microalgal transformation. (B) Real-time RT-PCR analysis. A total of $10 \mu \mathrm{L}$ of PCR products were separated by electrophoresis and gel image are shown. (C) Flow cytometry analysis of transgenic lines. A vertical dashed line is provided for visual reference. (D) Comparison of growth rates. Numbers indicated independent transgenic lines; WT, wild-type. (E) Confocal microscopic imaging of C. reinhardtii cells expressing mCherry-ATG8. Under normal growth condition, mCherry-ATG8 (red) diffused throughout the cytoplasm in transgenic cells. Upon autophagy induction by rapamycin $(500 \mathrm{nM})$ treatment for $16 \mathrm{~h}$, mCherry-ATG8 labeled vesicles appeared as bright dots. No mCherry fluorescence was detected in wild-type cells, indicating the specificity of mCherry signal. Chlorophyll $a$ fluorescence (blue) serves as reference for cell size and morphology. Results are representative images of three replicates. Bars, $10 \mu \mathrm{m}$. PSAD Pro, PSAD promoter; NdeI or XbaI, restriction sites; RBCS2 intron1, first intron of RBCS2 gene in C. reinhardtii; $A P H V I I I$, paromomycin-resistance gene; $P S A D_{T e r}, P S A D$ terminator. 


\section{Results}

\subsection{Autophagic Responses in C. reinhardtii Transgenic Lines Expressing mCherry-ATG8}

To monitor autophagy flux in C. reinhardtii, stable transgenic lines expressing a red fluorescent protein (mCherry)-tagged ATG8 fusion construct were generated. For this, the nuclear codon-optimized $m$ Cherry gene was fused to the full-length CrATG8 gene at the $\mathrm{N}$ terminus [25]. The $m$ Cherry-ATG8 fusion construct was expressed in C. reinhardtii using pChlamiRNA3int (pChl) expression system. This vector was designed for miRNA-mediated gene silencing in C. reinhardtii [26]. Herein, the artificial miRNA precursor was removed, and $m$ Cherry-ATG8 was cloned between PSAD promoter and PSAD terminator (see Materials and Methods section, Figure 1A). All putative transformants carrying the pChl-mCherry-ATG8 construct (hereafter referred to as mCherry-ATG8 transgenic lines) were confirmed by genomic PCR after several rounds of selection on paromomycin (APHVIII gene), and the expression of $m$ Cherry-ATG8 was analyzed using Real-time RT-PCR (Figure 1B). In addition, mCherry fluorescence intensity of transgenic lines were analyzed by flow cytometry (Figure 1C). Two transgenic lines (\#5 and \#8) showed the same growth characteristics from wild-type while exhibiting detectable levels of mCherry fluorescence (Figure 1B-D). Therefore, they were selected for further biochemical analyses, the pictures of transgenic line \#8 were presented in the study.

Confocal fluorescence microscope analyses revealed that mCherry-ATG8 diffused throughout the cytoplasm in cells growing under optimal conditions but appeared as bright dots after autophagy was induced by rapamycin treatment. No background fluorescence was detected in wild-type cells at the excitation/emission wavelengths of mCherry, indicating the specificity of the mCherry signal (Figure 1E). Although further confirmation would be necessary, these vesicles would likely correspond to autophagosomal structures, as rapamycin has been proven to induce the formation of autophagosomes in various species $[27,28]$.

It is a concern that both native ATG8 and the fluorescence-ATG8 fusion protein resulted from ectopic expression tend to be aggregated into intracellular inclusion bodies [29]. Thus, punctate structures positive with ATG8 or fluorescent-tagged ATG8 may correspond to either aggregates or functional autophagosomes [29]. To verify the nature of mCherry-ATG8 structures in this study, transgenic cells were examined by fluorescent confocal microscopy after $12 \mathrm{~h}$ of nitrogen starvation in the presence or absence of CQ (Figure 2). It has been shown that CQ inhibits autophagy by neutralizing the lysosomal/vacuolar $\mathrm{pH}$, thereby blocking the activity of hydrolytic enzymes and the subsequent steps in autophagy [22]. While concentrations up to $100 \mu \mathrm{M}$ of CQ did not affect cell viability, the ATG8 protein was more abundant and the lipidated form ATG8-PE was detectable in cells treated with $100 \mu \mathrm{M}$ CQ, indicating that a dose of $100 \mu \mathrm{M}$ CQ was sufficient to inhibit autophagic flux in C. reinhardtii (Figure 2A).

To investigate the role of lytic vacuoles in autophagic degradation in this alga, LysoSensor Green DND-189 (LSG) dye, a fluorescent probe that targets lysosomes/lytic vacuoles in various cell types, was used [30]. Flow cytometry analysis revealed a marked increase in LSG fluorescent intensity from $21.4 \pm 1.7$ arbitrary units (A.U.) of control (Rapamycin-/CQ-) to $36.5 \pm 7.3$ A.U. of rapamycin-treated cells (Rapamycin+/CQ-), corresponding to an increased formation of acidic lytic vacuoles (Figure 2B). This result is consistent with a previous research showing that rapamycin treatment triggered autophagy, leading to the accumulation of lytic vacuoles in C. reinhardtii [10]. In contrast to many vacuolar marker probes, LSG is pH sensitive and only exhibits fluorescence in acidic compartments [30]. As expected, treatment with CQ resulted in a reduced LSG fluorescent intensity $(20.9 \%$ and $33.1 \%$ of control and rapamycin-treated cells, respectively) (Figure 2B). These results indicated that LSG and CQ can be used to assess the vacuolar status in C. reinhardtii, and treatment with CQ caused a shift in vacuolar $\mathrm{pH}$ in this alga. In addition, Western blot analysis revealed a significant increase in ATG8-PE level in cells co-treated with rapamycin and CQ (Figure 2B), which confirmed that CQ can effectively inhibit autophagic degradation in C. reinhardtii [15]. 
A
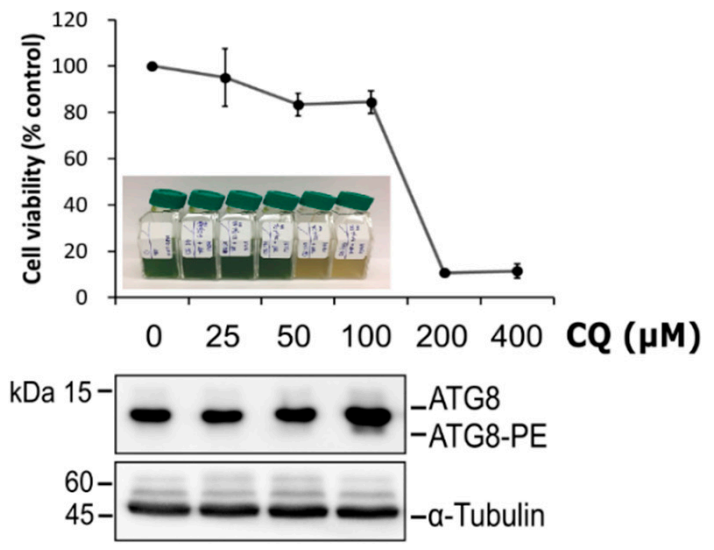

B

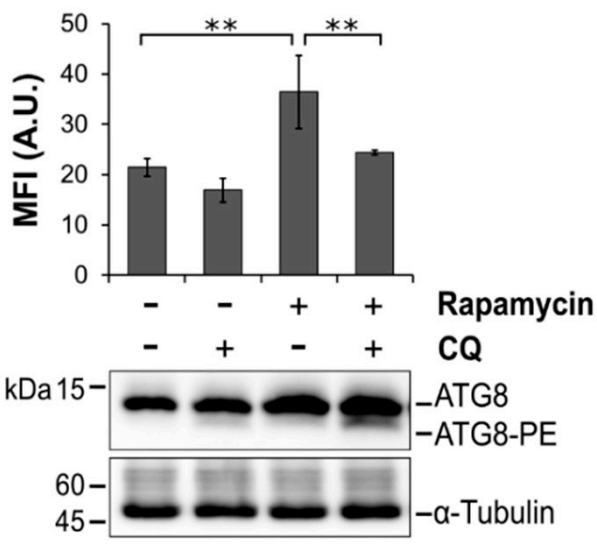

C
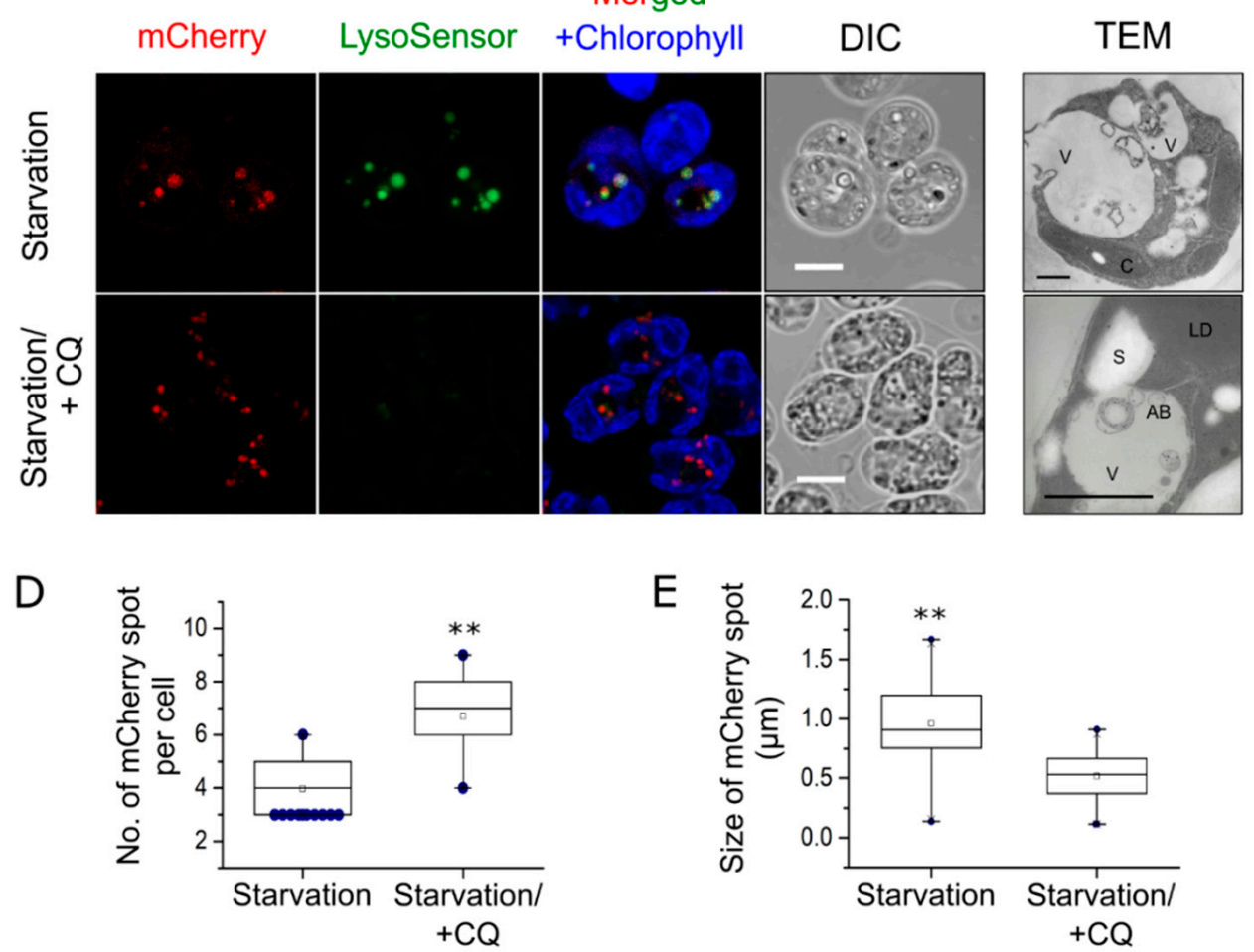

Figure 2. Influence of chloroquine on vacuolar acidification and autophagy activity in C. reinhardtii. (A) Cell viability and the corresponding ATG8 protein levels in C. reinhardtii cells treated with different concentrations of chloroquine (CQ) for $48 \mathrm{~h}$. The Western blot data for cells treated with $200 \mu \mathrm{M}$ and $400 \mu \mathrm{M} C Q$ are not available due to extensive cell death. The unconjugated form ATG8 and conjugated/lipidated form ATG8-PE are indicated. $\alpha$-Tubulin are shown as a loading control. (B) Mean fluorescence intensity (MFI) of LysoSensor Green DND-189 (LSG) and Western blot analysis of ATG8 in cells treated with rapamycin in the absence (-) or presence $(+)$ of $100 \mu \mathrm{M}$ CQ for $16 \mathrm{~h}$. A total of 100,000 cells were acquired for flow cytometry analysis per replicate per condition. (C) Co-localization of autophagosomes and lytic vacuoles. Cells expressing mCherry-ATG8 were subjected to nitrogen starvation for $12 \mathrm{~h}$ in the absence or presence of $100 \mu \mathrm{M} \mathrm{CQ}$, followed by staining with LSG. mCherry-ATG8 (red), LSG (green), and merged channels of mCherry-ATG8, LSG, and chlorophyll $a$ (blue) are shown. (D,E) Quantification of the number (D) or size (E) of structures labeled with mCherry in cells treated as in (C). Box plots indicate the medians, means, and quartiles while filled dots represent the outliers of each data set. Statistical significance was analyzed using one-way ANOVA and subsequent Tukey's post-hoc $t$ test and $P$-values obtained are indicated $(* *, p<0.01)$. Bars, $5 \mu \mathrm{m}$. 
To determine whether mCherry-ATG8 labeled autophagosomes in a specific manner, transgenic cells were subjected to nitrogen starvation to trigger autophagy and the distribution of mCherry-labeled structures was observed (Figure 2C). Upon nitrogen starvation, concurrent LSG staining revealed several enlarged yellow dots, which labeled with both mCherry and LSG, indicating that these structures are likely 'microalgal autolysosomes', a fusion compartment between autophagosome and lytic vacuole (Figure 2C, upper panels). The mCherry-labeled structures in this condition were relatively larger but fewer in number compared to those in the presence of CQ (Figure 2C-E). On the contrary, mCherry-labeled structures accumulated more numerously and had a less distinct size when vacuolar degradation was inhibited by $C Q$. These observations revealed dynamic changes in cellular distribution of mCherry-ATG8, thus, indicating that this fusion protein may constitute a good marker for the visualization of autophagic structures in C. reinhardtii. Overall, the present study provides evidences that autophagy is integrated with stress responses and the lytic vacuole could serve as the last destination for autophagic degradation in this microalga.

\subsection{Live Cell Imaging Revealed Interactions between Autophagosomes and Lipid Droplets in C. reinhardtii}

Algae usually tend to accumulate neutral lipids in a distinctive organelle called lipid droplet upon exposure to various stress conditions, especially nitrogen starvation. Under such conditions, autophagy is also induced, allowing the recycling of cellular components to support cell survival [15,31,32]. Although autophagy has been linked to the degradation of lipid droplets through a process termed lipophagy in mammals and yeast, the role of autophagy in lipid metabolism in microalgae remained to be explored. Herein, we investigated the potential interplay between autophagosomes and lipid droplets in C. reinhardtii using the mCherry-ATG8 marker. Live imaging revealed dynamic interactions between mCherry-labeled structures and lipid droplets (stained with BODIPY 505/515) throughout nitrogen starvation (Figure 3, Supplementary Videos S1-3). In the early stage of stress (first $12 \mathrm{~h}$ ), the newly formed autophagosomes were often found alongside the lipid droplets (Figure 3A, Supplementary Video S1). TEM analysis was used in an attempt to clarify the autophagosomes-lipid droplets interaction in the early stage of starvation [33]. As shown in Figure 3B, double-membrane vesicles that may correspond to autophagosomes were seen next to a lipid droplet in cells starved for $12 \mathrm{~h}$. It is interesting to note that we could not observe any fusion events between autophagosomes and lipid droplets in starved cells at $12 \mathrm{~h}$ post-starvation. The interaction between these two organelles at this stage remained to be explored, although it has been reported that normal autophagic flux is required for the formation of lipid droplets in C. reinhardtii $[15,16]$.

In addition to the above findings, we detected a few punctate structures labeled with both mCherry-ATG8 and BOPIDY after prolonged periods of starvation $(24 \mathrm{~h}$ ) (Supplementary Video S3). The number of yellow (red + green) punctates was significantly increased in starved cells at $48 \mathrm{~h}$ post-starvation (Figure 3C). Confocal microscopic image of a C. reinhardtii cell expressing mCherry-ATG8 starved for $48 \mathrm{~h}$ (Figure 3D). The fluorescence intensity profiles taken along the dashed lines indicated distinct phases of autophagosome-lipid droplet fusion (Figure 3E,F). For example, a lipid droplet (green) can be easily distinguished with an autophagosome-lipid droplet fusion (yellow) or an autophagosome (red) based on their intensity profiles. A three-dimensional (3D) rendering from Z-stacks of the cell in Figure 3D is available in Supplementary Video S2. Taken together, these data suggested that autophagosomes might be in association with microalgal lipid droplets through a process similar to lipophagy, indicating by the fusion between autophagosomes and lipid droplets at the later stages of nitrogen starvation. It remains to be explored how physical interaction occurs between autophagosomes and lipid droplets, and the relative contribution of autophagy-related pathway versus lipase-mediated degradation of lipid droplets in microalgae. 

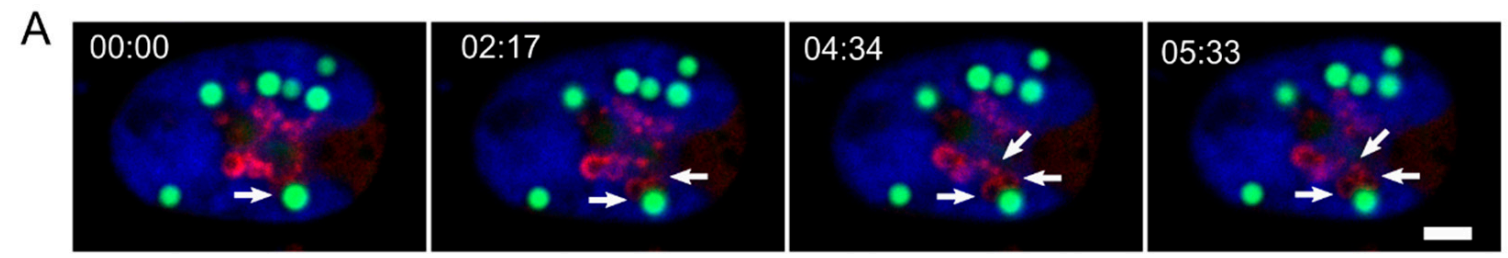

B
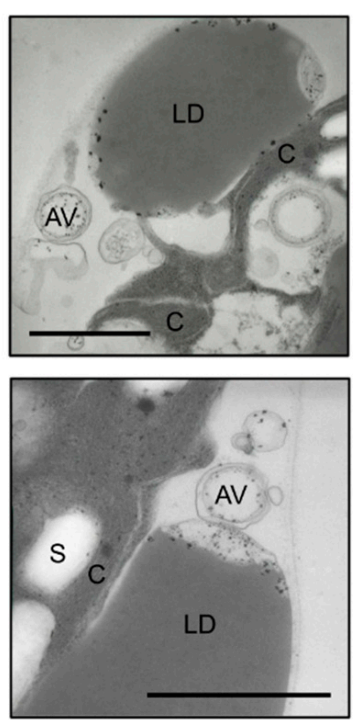

C

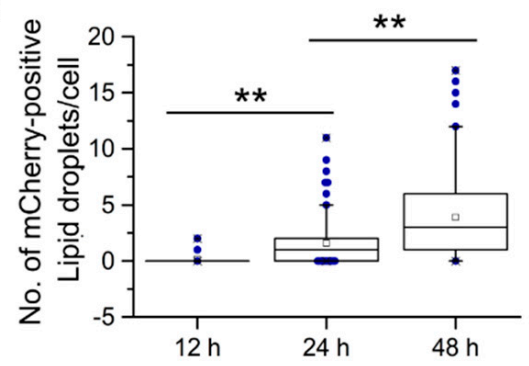

D

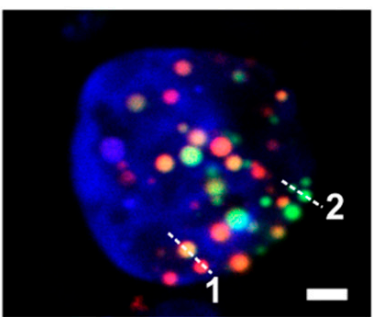

$\mathrm{E}$

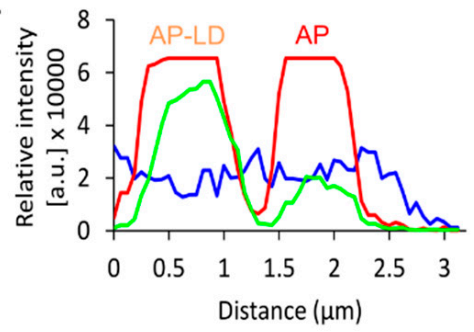

$\mathrm{F}$

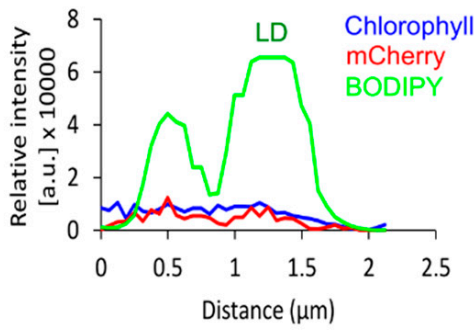

Figure 3. Dynamic interactions between autophagosome and lipid droplets in C. reinhardtii. (A) Still frames taken from Supplementary Video S1 showed that mCherry-ATG8 labeled structures were in close proximity to a lipid droplet (LD) in algal cells starved for nitrogen for $12 \mathrm{~h}$. (B) TEM images of cells grown under same condition as in (A) showing autophagic vacuoles near LDs. (C) Quantification of LD positive with mCherry-ATG8 at $12 \mathrm{~h}, 24 \mathrm{~h}$, and $48 \mathrm{~h}$ of nitrogen starvation. The number of vesicles labeled with both red and green fluorescence was counted per cell (50-80 cells per condition) using the Co-localization plugin (ImageJ). Box plots indicate the medians, means and quartiles. Outliers of each data set are presented as filled dots. Statistical significance was analyzed using the Mann-Whitney $\mathrm{U}$ test and P-values obtained are indicated $(* *, p<0.01)$. (D) Representative image of a cell starved for 48 h. mCherry-ATG8 (red), BODIPY 505/515 (green), and merged channels of mCherry-ATG8, BODIPY, and chlorophyll $a$ (blue) are shown. (E,F) Fluorescence intensity profiles along the dashed line 1-2 in (D), respectively. Bars, $2 \mu \mathrm{m}$ in (A,D) or $1 \mu \mathrm{m}$ in (B). AV, autophagic vacuole; LD, lipid droplet; $\mathrm{C}$, chloroplast; $\mathrm{S}$, starch granule; AP-LD, fusion between an autophagosome and a lipid droplet; $\mathrm{AP}$, autophagosome.

\section{Discussion}

In recent years, studies of autophagy in photosynthetic organisms including land plants and microalgae have been greatly expanded. Recent findings suggested that autophagy might play a pivotal role in triacylglycerols biosynthesis and lipid droplets formation in C. reinhardtii $[15,16]$. However, there is no confirmatory evidence on the same as well as the role of lipophagy in lipid turnover in microalgae [19]. By using the mCherry-ATG8 marker, we were able to visualize the autophagy process in living C. reinhardtii cells grown under various conditions.

It is now known that plants may have distinct types of selective autophagy which might not be conserved in non-photosynthetic organisms [34]. Since C. reinhardtii is a model organism that retains important features of both plants and animals, a better understanding of autophagy in this alga would constitute the missing link between these two eukaryotic kingdoms [35,36]. Previous works have tentatively suggested that endogenous ATG8 diffusely distributes in the cytoplasm of $C$. reinhardtii cells under ideal conditions but relocates to punctate structures that might represent autophagosomes upon the induction of autophagy [9]. Similar localization patterns were observed in the present study 
in C. reinhardtii using the mCherry-ATG8 marker. The degradation of autophagosome and its cargo has been known to occur inside the autolysosome, a fused structure between an autophagosome and a lysosome containing hydrolytic enzymes, in animal cells [37]. By using TEM imaging, a previous study has shown that autophagic bodies are accumulated within the vacuoles in C. reinhardtii cells with impaired lytic function [15]. In this study, we indeed observed significant colocalization of lytic vacuoles with mCherry-labeled autophagosomes under nitrogen starvation, confirming that the vacuoles are involved in autophagic degradation in this alga (Figure 2).

Over the last two decades, lipid droplets have received intensive focus [38]. Lipid droplets not only serve as storage depots for cellular lipids, but also participate in multiple physiological pathways, including protein storage, membrane transport and the replication of pathogenic viruses [39]. The breakdown of neutral lipids core in lipid droplets is primarily accomplished by lipolysis, which involves a variety of cytosolic lipases [39]. In addition to the lipolysis pathway, recent studies have indicated the participation of autophagy in lipid droplet turnover through a process known as lipophagy in mouse, rice, yeast, and microalgae [19,40-44]. By using mCherry-ATG8 tool, we confirm the physical interactions between autophagosomes and lipid droplets in C. reinhardtii (Figure 3). At the early stage of starvation, autophagy may supply precursors for lipid droplets biogenesis in microalgae through an unexplored mechanism, as we observed that autophagosomes maintained close contact but did not fuse to lipid droplets. Indeed, nutrient recycling via autophagy has been reported to play a crucial role in the regeneration of precursors for the formation of lipid droplets in C. reinhardtii $[15,16]$. Recently, autophagy has been shown to play a dual role in controlling lipid synthesis and degradation in the model plant Arabidopsis thaliana [45]. It would be interesting to explore the interplay between autophagosomes and lipid droplets in microalgae. The fusion of autophagosomes and lipid droplets at the later stages of starvation suggests the involvement of an autophagic pathway in the breakdown of lipid droplets in C. reinhardtii to sustain cellular homeostasis and promote cell survival. It remains to be elucidated how autophagosomes sequester lipid droplets in this alga. Taken together, it is likely that under stress conditions, the formation and degradation of both autophagosomes and lipid droplets occur in a hierarchical and balanced fashion in microalgae. Thus, understanding the molecular mechanism between autophagy and lipid metabolism will open up new avenues of biofuels production from these unicellular organisms.

Supplementary Materials: The following are available online at http://www.mdpi.com/2073-4409/8/9/992/s1, Video S1: Autophagosomes localized in close apposition with a lipid droplet in C. reinhardtii cells starved for nitrogen for $12 \mathrm{~h}$. mCherry-ATG8 (red), BODIPY 505/515 (green), and merged channels of mCherry-ATG8 and BODIPY are shown. Within the first $12 \mathrm{~h}$ of nitrogen starvation, the newly formed autophagosomes were often found alongside the lipid droplets; Video S2: Three-dimensional (3D) rendering from Z-stack images of a representative C. reinhardtii cell starved for nitrogen for $48 \mathrm{~h}$. Autophagosomes were labeled in red color while lipid droplets were labeled in green color. Fused structures between autophagosomes and lipid droplets (red + green) can be observed throughout cellular cytoplasm; Video S3: Co-localization of mCherry-ATG8 labeled autophagosomes and lipid droplets in C. reinhardtii cells after $24 \mathrm{~h}$ of nitrogen starvation. mCherry-ATG8 (red), BODIPY 505/515 (green), and merged channels of mCherry-ATG8, BODIPY, and chlorophyll $a$ (blue) are shown.

Author Contributions: Q.-G.T., R.R., and H.-S.K. conceived the research plan; Q.-G.T. performed most of the experiments and analyzed the data; H.R.Y. performed confocal and FACS analysis of the mutants; R.R. performed TEM analysis; H.-S.K. supervised the project; J.L.C. contributed anti-CrATG8 antibody and technical assistance to Western blot analysis; S.-J.L., R.R., and K.C. contributed to the interpretation of the results; Q.-G.T. and H.-S.K. wrote the article with contributions of all the authors.

Funding: This research was funded by a grant from Marine Biotechnology Program (20150184) funded by Ministry of Oceans and Fisheries, Korea; by the Advanced Biomass R\&D Center (ABC) of Global Frontier Project funded by the Ministry of Science and ICT(ABC-2015M3A6A2065697); and by grant from the Korea Research Institute of Bioscience and Biotechnology (KRIBB) Research Initiative Program (www.kribb.re.kr).

Conflicts of Interest: The authors declare no conflict of interest. 


\section{References}

1. Minhas, A.K.; Hodgson, P.; Barrow, C.J.; Adholeya, A. A Review on the Assessment of Stress Conditions for Simultaneous Production of Microalgal Lipids and Carotenoids. Front. Microbiol. 2016, 7, 546-565. [CrossRef]

2. Cho, D.-H.; Ramanan, R.; Heo, J.; Shin, D.-S.; Oh, H.-M.; Kim, H.-S. Influence of limiting factors on biomass and lipid productivities of axenic Chlorella vulgaris in photobioreactor under chemostat cultivation. Bioresour. Technol. 2016, 211, 367-373. [CrossRef]

3. Kakarla, R.; Choi, J.-W.; Yun, J.-H.; Kim, B.-H.; Heo, J.; Lee, S.; Cho, D.-H.; Ramanan, R.; Kim, H.-S. Application of high-salinity stress for enhancing the lipid productivity of Chlorella sorokiniana HS1 in a two-phase process. J. Microbiol. 2018, 56, 56-64. [CrossRef]

4. Cho, K.; Cho, D.-H.; Heo, J.; Kim, U.; Lee, Y.J.; Choi, D.-Y.; Kim, H.-S. Nitrogen modulation under chemostat cultivation mode induces biomass and lipid production by Chlorella vulgaris and reduces antenna pigment accumulation. Bioresour. Technol. 2019, 281, 118-125. [CrossRef]

5. Kroemer, G.; Mariño, G.; Levine, B. Autophagy and the integrated stress response. Mol. Cell 2010, 40, $280-293$. [CrossRef]

6. Bassham, D.C.; Laporte, M.; Marty, F.; Moriyasu, Y.; Ohsumi, Y.; Olsen, L.J.; Yoshimoto, K. Autophagy in development and stress responses of plants. Autophagy 2006, 2, 2-11. [CrossRef]

7. Glick, D.; Barth, S.; Macleod, K.F. Autophagy: Cellular and molecular mechanisms. J. Pathol. 2010, $221,3-12$. [CrossRef]

8. Michaeli, S.; Galili, G.; Genschik, P.; Fernie, A.R.; Avin-Wittenberg, T. Autophagy in Plants-What's New on the Menu? Trends Plant Sci. 2015, 21, 134-144. [CrossRef]

9. Pérez-Pérez, M.; Couso, I.; Heredia-Martínez, L.; Crespo, J. Monitoring Autophagy in the Model Green Microalga Chlamydomonas reinhardtii. Cells 2017, 6, 36. [CrossRef]

10. Crespo, J.L.; Díaz-Troya, S.; Florencio, F.J. Inhibition of Target of Rapamycin Signaling by Rapamycin in the Unicellular Green Alga Chlamydomonas reinhardtii. Plant Physiol. 2005, 139, 1736-1749. [CrossRef]

11. Pérez-Pérez, M.E.; Florencio, F.J.; Crespo, J.L. Inhibition of Target of Rapamycin Signaling and Stress Activate Autophagy in Chlamydomonas reinhardtii. Plant Physiol. 2010, 152, 1874-1888. [CrossRef]

12. Pérez-Pérez, M.E.; Couso, I.; Crespo, J.L. Carotenoid deficiency triggers autophagy in the model green alga Chlamydomonas reinhardtii. Autophagy 2012, 8, 376-388. [CrossRef]

13. Pérez-Martín, M.; Pérez-Pérez, M.E.; Lemaire, S.D.; Crespo, J.L. Oxidative Stress Contributes to Autophagy Induction in Response to Endoplasmic Reticulum Stress in Chlamydomonas reinhardtii. Plant Physiol. 2014, 166, 997-1008. [CrossRef]

14. Pérez-Martín, M.; Blaby-Haas, C.E.; Pérez-Pérez, M.E.; Andrés-Garrido, A.; Blaby, I.K.; Merchant, S.S.; Crespo, J.L. Activation of Autophagy by Metals in Chlamydomonas reinhardtii. Eukaryot. Cell 2015, 14, 964-973. [CrossRef]

15. Couso, I.; Pérez-Pérez, M.E.; Martínez-Force, E.; Kim, H.-S.; He, Y.; Umen, J.G.; Crespo, J.L.; Bozhkov, P. Autophagic flux is required for the synthesis of triacylglycerols and ribosomal protein turnover in Chlamydomonas. J. Exp. Bot. 2017, 69, 1355-1367. [CrossRef]

16. Pugkaew, W.; Meetam, M.; Ponpuak, M.; Yokthongwattana, K.; Pokethitiyook, P. Role of autophagy in triacylglycerol biosynthesis in Chlamydomonas reinhardtii revealed by chemical inducer and inhibitors. J. Appl. Phycol. 2017, 30, 15-22. [CrossRef]

17. Ramanan, R.; Tran, Q.-G.; Cho, D.-H.; Jung, J.-E.; Kim, B.-H.; Shin, S.-Y.; Choi, S.-H.; Liu, K.-H.; Kim, D.-S.; Lee, S.-J.; et al. The Ancient Phosphatidylinositol 3-Kinase Signaling System Is a Master Regulator of Energy and Carbon Metabolism in Algae. Plant Physiol. 2018, 177, 1050-1065. [CrossRef]

18. Tran, Q.-G.; Cho, K.; Kim, U.; Yun, J.-H.; Cho, D.-H.; Heo, J.; Park, S.-B.; Kim, J.W.; Lee, Y.J.; Ramanan, R.; et al. Enhancement of $\beta$-carotene production by regulating the autophagy-carotenoid biosynthesis seesaw in Chlamydomonas reinhardtii. Bioresour. Technol. 2019, 292, 121937. [CrossRef]

19. Zhao, L.; Dai, J.; Wu, Q. Autophagy-like processes are involved in lipid droplet degradation in Auxenochlorella protothecoides during the heterotrophy-autotrophy transition. Front. Plant Sci. 2014, 5, 400. [CrossRef]

20. Mizushima, N.; Yoshimori, T.; Levine, B. Methods in mammalian autophagy research. Cell 2010, 140, $313-326$. [CrossRef] 
21. Nakatogawa, H.; Ichimura, Y.; Ohsumi, Y. Atg8, a ubiquitin-like protein required for autophagosome formation, mediates membrane tethering and hemifusion. Cell 2007, 130, 165-178. [CrossRef]

22. Klionsky, D.J.; Abdelmohsen, K.; Abe, A.; Abedin, M.J.; Abeliovich, H.; Acevedo-Arozena, A.; Adachi, H.; Adams, C.M.; Adams, P.D.; Adeli, K.; et al. Guidelines for the use and interpretation of assays for monitoring autophagy (3rd edition). Autophagy 2016, 12, 1-222. [CrossRef]

23. Harris, E.H. Chapter 8-Chlamydomonas in the Laboratory. In The Chlamydomonas Sourcebook, 2nd ed.; Harris, E.H., Stern, D.B., Witman, G.B., Eds.; Academic Press: London, UK, 2009; pp. 241-302.

24. Rasala, B.A.; Barrera, D.J.; Ng, J.; Plucinak, T.M.; Rosenberg, J.N.; Weeks, D.P.; Oyler, G.A.; Peterson, T.C.; Haerizadeh, F.; Mayfield, S.P. Expanding the spectral palette of fluorescent proteins for the green microalga Chlamydomonas reinhardtii. Plant. J. 2013, 74, 545-556. [CrossRef]

25. Klionsky, D.J. For the last time, it is GFP-Atg8, not Atg8-GFP (and the same goes for LC3). Autophagy 2011, 7, 1093-1094. [CrossRef]

26. Molnar, A.; Bassett, A.; Thuenemann, E.; Schwach, F.; Karkare, S.; Ossowski, S.; Weigel, D.; Baulcombe, D. Highly specific gene silencing by artificial microRNAs in the unicellular alga Chlamydomonas reinhardtii. Plant. J. 2009, 58, 165-174. [CrossRef]

27. Pérez-Pérez, M.E.; Couso, I.; Crespo, J.L. The TOR Signaling Network in the Model Unicellular Green Alga Chlamydomonas reinhardtii. Biomolecules 2017, 7, 54. [CrossRef]

28. Crespo, J.L.; Hall, M.N. Elucidating TOR Signaling and Rapamycin Action: Lessons from Saccharomyces cerevisiae. Microbiol. Mol. Biol. Rev. 2002, 66, 579-591. [CrossRef]

29. Kuma, A.; Matsui, M.; Mizushima, N. LC3, an autophagosome marker, can be incorporated into protein aggregates independent of autophagy: Caution in the interpretation of LC3 localization. Autophagy 2007, 3, 323-328. [CrossRef]

30. Galindo, F.; Burguete, M.I.; Vigara, L.; Luis, S.V.; Kabir, N.; Gavrilovic, J.; Russell, D.A. Synthetic macrocyclic peptidomimetics as tunable $\mathrm{pH}$ probes for the fluorescence imaging of acidic organelles in live cells. Angew. Chem. Int. Ed. 2005, 117, 6662-6666. [CrossRef]

31. Gao, Q.; Goodman, J.M. The lipid droplet-A well-connected organelle. Front. Cell Dev. Biol. 2015,3 , 49. [CrossRef]

32. Tran, Q.-G.; Cho, K.; Park, S.-B.; Kim, U.; Lee, Y.J.; Kim, H.-S. Impairment of starch biosynthesis results in elevated oxidative stress and autophagy activity in Chlamydomonas reinhardtii. Sci. Rep. 2019, 9, 9856. [CrossRef] [PubMed]

33. Singh, R.; Kaushik, S.; Wang, Y.; Xiang, Y.; Novak, I.; Komatsu, M.; Tanaka, K.; Cuervo, A.M.; Czaja, M.J. Autophagy regulates lipid metabolism. Nature 2009, 458, 1131. [CrossRef] [PubMed]

34. Bozhkov, P.V. Plant autophagy: Mechanisms and functions. J. Exp. Bot. 2018, 69, 1281-1285. [CrossRef] [PubMed]

35. Merchant, S.S.; Prochnik, S.E.; Vallon, O.; Harris, E.H.; Karpowicz, S.J.; Witman, G.B.; Terry, A.; Salamov, A.; Fritz-Laylin, L.K.; Maréchal-Drouard, L.; et al. The Chlamydomonas Genome Reveals the Evolution of Key Animal and Plant Functions. Science 2007, 318, 245-250. [CrossRef] [PubMed]

36. Gutman, B.L.; Niyogi, K.K. Chlamydomonas and Arabidopsis. A Dynamic Duo. Plant Physiol. 2004, 135, 607-610. [CrossRef] [PubMed]

37. Nagy, P.; Varga, Á.; Kovács, A.L.; Takáts, S.; Juhász, G. How and why to study autophagy in Drosophila: It's more than just a garbage chute. Methods 2015, 75, 151-161. [CrossRef] [PubMed]

38. Thiam, A.R.; Farese, R.V., Jr.; Walther, T.C. The biophysics and cell biology of lipid droplets. Nat. Rev. Mol. Cell Biol. 2013, 14, 775-786. [CrossRef]

39. Walther, T.C.; Farese, R.V., Jr. Lipid droplets and cellular lipid metabolism. Annu. Rev. Biochem. 2012, 81, 687-714. [CrossRef]

40. Singh, R.; Cuervo, A.M. Lipophagy: Connecting Autophagy and Lipid Metabolism. Int. J. Cell Biol. 2012, 2012, 282041. [CrossRef]

41. Kurusu, T.; Koyano, T.; Hanamata, S.; Kubo, T.; Noguchi, Y.; Yagi, C.; Nagata, N.; Yamamoto, T.; Ohnishi, T.; Okazaki, Y. OsATG7 is required for autophagy-dependent lipid metabolism in rice postmeiotic anther development. Autophagy 2014, 10, 878-888. [CrossRef]

42. Van Zutphen, T.; Todde, V.; de Boer, R.; Kreim, M.; Hofbauer, H.F.; Wolinski, H.; Veenhuis, M.; van der Klei, I.J.; Kohlwein, S.D. Lipid droplet autophagy in the yeast Saccharomyces cerevisiae. Mol. Biol. Cell 2014, 25, $290-301$. [CrossRef] [PubMed] 
43. Dong, H.; Czaja, M.J. Regulation of lipid droplets by autophagy. Trends Endocrinol. Metab. 2011, 22, $234-240$. [CrossRef] [PubMed]

44. Liu, K.; Czaja, M.J. Regulation of lipid stores and metabolism by lipophagy. Cell Death Differ. 2012, $20,3$. [CrossRef] [PubMed]

45. Fan, J.; Yu, L.; Xu, C. Dual Role for Autophagy in Lipid Metabolism in Arabidopsis. Plant Cell 2019, 31 , 1598-1613. [CrossRef] [PubMed]

(C) 2019 by the authors. Licensee MDPI, Basel, Switzerland. This article is an open access article distributed under the terms and conditions of the Creative Commons Attribution (CC BY) license (http://creativecommons.org/licenses/by/4.0/). 